\title{
An agent-based model of muscle contraction process as a bio-robotic process
}

\begin{abstract}
This paper introduces a new computational methodology to model muscle contraction process as a bio-robotic process using agent technology. In this work, we have focused on muscle myosin nanomotor as the driven motor of muscles and introduced the nanomotor as a physical intelligent agent. Then, the mechanism of the nanomotor was specified using subsumption architecture of agent technology and modeled with the Finite State Machine (FSM) diagram of Unified Modeling Language (UML). The proposed agent-based FSM model of the mechanism of muscle myosin nanomotor illustrated the internal intelligent and autonomous decision-making process of the nanomotor as a robot mechanism. In order to verify the proposed agent-based FSM model of the mechanism of the nanomotor, we developed its mathematical definitions (its Deterministic Finite Automaton (DFA) and grammar) and compared them with the natural behavior of the nanomotor inside the muscle cells. The comparison results indicated that the mechanism of muscle myosin nanomotor could be defined as a robot mechanism with its inputs, internal decision-making process, and outputs. As muscle contraction process is a set of the mechanisms of muscle myosin nanomotors, our proposed agent-based model of the mechanism of the nanomotor can introduce muscle contraction process as a general bio-robotic process.
\end{abstract}

Keyword: Agent technology; Bio-robotic system; Muscle contraction process; Muscle myosin nanomotor; UML 